\title{
Assessment of the main pathogens associated with clinical and subclinical endometritis in cows by culture and MALDI-TOF mass spectrometry identification
}

\author{
R. B. Paiano, ${ }^{1 *}$ L. Z. Moreno, ${ }^{2} \odot$ V. T. M. Gomes, ${ }^{2}$ B. M. Parra, ${ }^{2} \odot$ M. R. Barbosa, ${ }^{3} \oplus$ M. I. Z. Sato, ${ }^{3}$ J. Bonilla, ${ }^{4}$ \\ G. Pugliesi, ${ }^{1}$ (D) P. S. Baruselli, ${ }^{1}$ (D) and A. M. Moreno ${ }^{2 *}$ (I) \\ ${ }^{1}$ Department of Animal Reproduction, School of Veterinary Medicine and Animal Science, University of São Paulo, São Paulo, SP 05508270, \\ Brazil \\ ${ }^{2}$ Department of Preventive Veterinary Medicine and Animal Health, School of Veterinary Medicine and Animal Science, University of São Paulo, \\ São Paulo, SP 05508 270, Brazil \\ ${ }^{3}$ Environmental Company of the State of São Paulo (CETESB), São Paulo, SP 05459-900, Brazil \\ ${ }^{4}$ Department of Food Engineering, College of Animal Science and Food Engineering, University of São Paulo, Pirassununga, SP 13635900, Brazil
}

\section{ABSTRACT}

Clinical endometritis (CE) and subclinical endometritis (SCE) are diseases that affect dairy cows during the puerperium, causing negative effects on the animals' milk production and fertility. The objective of this study was to assess the main bacteria related to cases of $\mathrm{CE}$ and SCE from uterine samples of dairy cows in Brazilian herds. Selective and differential media were used for isolation of aerobic and anaerobic bacteria and further MALDI-TOF mass spectrometry (MS) identification. A total of 279 lactating dairy cows with 28 to $33 \mathrm{~d}$ in milk from 6 commercial farms were evaluated. Initially, cows were classified in 3 groups: cytologic healthy cows $(\mathrm{n}=161)$, cows with CE (n $=83)$, and cows with SCE $(\mathrm{n}=35)$. Healthy animals presented 97 species, followed by the $\mathrm{CE}$ group with 53 identified species, and SCE cows presented only 21 bacterial species. We found a significantly higher isolation rate of Trueperella pyogenes in $\mathrm{CE}$ (26.5\%) cows compared with healthy and SCE cows. Some anaerobic species were exclusively isolated from the CE group, even though they presented lower frequency. Interestingly, $18.1 \%$ of samples from CE cows and $40 \%$ of SCE cows were negative to bacterial isolation. Despite the use of culture-dependent methods instead of molecular methods, the present study enabled the identification of a complex community of 127 different species from 48 genera, composed of aerobic and anaerobic bacterial species among the 3 different animal groups. The method of sample collection, culture, and identification

\footnotetext{
Received April 21, 2021.

Accepted January 3, 2022.

*Corresponding authors: renanpaiano@hotmail.com and morenoam@usp.br
}

by MALDI-TOF MS were essential for the success of the analyses.

Key words: clinical endometritis, subclinical endometritis, uterine microbiota, dairy cow, matrixassisted laser desorption/ionization time-of-flight mass spectrometry

\section{INTRODUCTION}

Endometritis is one of the most important causes of infertility in dairy herds. Its effects on dairy farms include the costs related to the treatment of the affected animals, milk discard, culling, decrease in milk production, and worsened fertility of the affected cows (Gilbert et al., 2005; LeBlanc, 2008; Paiano et al., 2019, 2020a,b).

Endometritis is the inflammation of the endometrium, the innermost lining of the uterus, being subdivided into clinical endometritis $(\mathbf{C E})$ and subclinical endometritis (SCE; Sheldon et al., 2006). Clinical endometritis is defined as the presence of purulent or mucopurulent vaginal discharge detected $3 \mathrm{wk}$ or more postpartum (LeBlanc et al., 2002). Subclinical endometritis is characterized by an increased proportion of $\mathrm{PMN}$ in the endometrium in the absence of clinical disease (Gilbert et al., 2005).

Previous studies have reported that the main bacterial species associated with endometritis in dairy cows are Trueperella pyogenes, Escherichia coli, and Fusobacterium necrophorum (Williams et al., 2005; Sheldon et al., 2010; Machado et al., 2012; Bicalho et al., 2017a; Bogado Pascottini et al., 2021). Currently, the main techniques described for the identification of uterine microorganisms involve culture-dependent methods or metagenomic analysis. When the studies involve culture and identification of isolated bacteria, this can 
be conducted using traditional biochemical methods or more modern techniques such as MALDI-TOF MS (Ballas et al., 2021), sequencing of 16S rRNA gene (Bogado Pascottini et al., 2020), or Fourier-transform infrared (Prunner et al., 2014; Wagener et al., 2014). Metagenomic analyses are conducted via $16 \mathrm{~S}$ rRNA gene profiling by high-throughput sequencing (Bicalho et al., 2017b; Wang et al., 2018).

Considering these points, the objective of this study was to identify the main bacteria present in cases of $\mathrm{CE}$ and SCE from uterine samples of dairy cows in Brazilian herds.

\section{MATERIALS AND METHODS}

\section{Study Farms}

The study was conducted between May and November 2019 on 6 commercial dairy farms in São Paulo State, Brazil. Lactating Holstein dairy cows were housed in freestall barns. Cows were fed twice daily with a TMR diet consisting of corn silage with cornmeal, soybean meal, and mineral supplements, formulated according to NRC (2001) to meet the nutritional requirements for lactating Holstein cows. Cows were milked twice daily. The voluntary waiting period was set at 40 to 45 d postpartum. This study was approved by the Ethics Committee for the Use of Animals of the School of Veterinary Medicine and Animal Science, University of São Paulo, São Paulo, Brazil, approval no. 1377270218.

\section{Sampling and Case Classification}

A total of 279 cows were examined. This was a convenience sample, of which the evaluated animals were originally sampled for the assessment of healthy and diseased individuals based on previously reported endometritis incidence of $23.8 \%$ and $95 \%$ confidence level with $5 \%$ precision (Thrusfield, 2005). Only cows that were not treated with antibiotics drugs or nonsteroidal or steroidal anti-inflammatory drugs after parturition were included in this study. In addition, the cows sampled in the present study showed no clinical signs of clinical mastitis, milk fever, or ketosis.

Endometrial cytobrush samples were collected between 28 and $33 \mathrm{~d}$ after parturition. Briefly, the perineal area of the cow was cleansed with $70 \%$ ethylic alcohol and dried using a paper towel. A sterile cytobrush rod (covered with a sterile sanitary sheath) was introduced into the vagina and guided through the cervix per rectum, as described by Bogado Pascottini et al. (2020); control swabs were not collected. After removal from the genital tract, the cytobrush was gently rolled onto a sterilized microscope slide. The first $5 \mathrm{~cm}$ of the cytobrush was then cut with sterile scissors, placed in a sterile $15-\mathrm{mL}$ plastic conical tube with anaerobic transport medium, and kept at $4^{\circ} \mathrm{C}$ until arrival at the laboratory for bacteriological examination.

After each cytobrush sampling, vaginal discharge was evaluated using a Metricheck device (Simcro Tech) and scored as $0=$ clear mucus, $1=$ mucus with flecks of pus, $2=$ mucopurulent discharge ( $\leq 50 \%$ pus $)$, and $3=$ purulent discharge ( $>50 \%$ pus). Cytology slides were stained using the panoptic rapid staining method (Laborclin, Pinhais, Brazil). Evaluations were performed under a microscope at $400 \times$ magnification (Nikon E200). A total of 200 cells were counted in the cytological slide to determine the proportion of PMN (Bogado Pascottini et al., 2020).

Animals were classified into 3 groups based on uterine health considering vaginal discharge and cytological analysis: healthy cows (without $\mathrm{CE}$ and without SCE, $\mathrm{n}=161$ ), cows with $\mathrm{CE}$ (vaginal discharge score $=3$ and $>18 \%$ PMN, $\mathrm{n}=83$ ), and cows with SCE (vaginal discharge 0,1 , or 2 , and $>18 \%$ PMN, $\mathrm{n}=35$; Kasimanickam et al., 2004). Four cows with vaginal discharge score $=3$ and PMN $<18$ that were considered to have cervicitis were not included in the study.

\section{Bacterial Isolation}

Immediately after sampling, the cytobrushes were transferred to $6 \mathrm{~mL}$ of anaerobic transport medium. At the laboratory, the cytobrushes were washed in $2 \mathrm{~mL}$ of Brucella broth (Difco-BBL). Then, $10 \mu \mathrm{L}$ of broth was streaked onto Brucella agar (Difco-BBL) with 7\% whole defibrinated horse blood, supplemented with hemin $(5 \mathrm{mg} / \mathrm{L})$ and menadione $(1 \mathrm{mg} / \mathrm{L})$, and plates were incubated at $37^{\circ} \mathrm{C}$ for $48 \mathrm{~h}$ under anaerobic conditions. An aliquot of $10 \mu \mathrm{L}$ of broth was also streaked onto MacConkey agar, CHROMagar Orientation, and blood agar with $5 \%$ defibrinated sheep blood (Difco$\mathrm{BBL}$ ). These plates were incubated at $37^{\circ} \mathrm{C}$ for 24 to $48 \mathrm{~h}$ under aerobic conditions. All morphologically different colonies were identified by MALDI-TOF MS and stored at $-86^{\circ} \mathrm{C}$ in brain-heart infusion medium (Difco) with $30 \%$ glycerol, supplemented with fetal calf serum $(5 \%)$.

Identification of the bacteria by MALDI-TOF MS was carried out as described previously by Hijazin et al. (2012). Mass spectra were acquired using a Microflex mass spectrometer (Bruker Daltonik) and identified with the manufacturer's software MALDI BioTyper 3.0. The requirements for interpreting the standards of the manufacturer Bruker Daltonik were used in this study as follows: scores $\geq 2.0$ were accepted for species 
Table 1. Bacterial population isolated from dairy cows with clinical (CE) and subclinical (SCE) endometritis; percentages shown in parentheses

\begin{tabular}{lccc}
\hline Bacteria & $\mathrm{CE}(\mathrm{n}=83)$ & $\mathrm{SCE}(\mathrm{n}=35)$ & Healthy $(\mathrm{n}=161)$ \\
\hline Trueperella pyogenes & $22(26.51)^{\mathrm{a}}$ & $0(0.0)^{\mathrm{b}}$ & $1(0.6)^{\mathrm{b}}$ \\
Escherichia coli & $16(19.3)$ & $2(5.7)$ & $24(14.9)$ \\
Bacillus spp. & $14(16.9)$ & $8(20.0)$ & $21(13.0)$ \\
CNS & $14(16.9)$ & $2(5.7)$ & $32(19.9)$ \\
Aerococcus viridans & $11(13.3)$ & $5(14.2)$ & $16(9.9)$ \\
Pseudomonas spp. & $7(8.4)$ & $4(10.0)$ & $8(5.0)$ \\
Enterococcus spp. & $6(7.2)$ & $0(0.0)$ & $19(11.8)$ \\
Helcococcus spp. & $5(6.0)$ & $0(0.0)$ & $0(0.0)$ \\
Q-Hemolytic streptococci & $5(6.0)$ & $3(7.5)$ & $9(5.6)$ \\
Paenibacillus spp. & $4(4.8)$ & $3(7.5)$ & $11(6.8)$ \\
Prevotella heparinolytica & $4(4.8)$ & $0(0.0)$ & $0(0.0)$ \\
Bacteroides pyogenes & $3(3.6)$ & $0(0.0)$ & $0(0.0)$ \\
Klebsiella spp. & $3(3.6)$ & $0(0.0)$ & $4(2.5)$ \\
Lysinibacillus spp. & $3(3.6)$ & $0(0.0)$ & $3(1.9)$ \\
Porphyromonas levii & $3(3.6)$ & $0(0.0)$ & $0(0.0)$ \\
Bifidobacterium pseudolongom & $2(2.4)$ & $0(0.0)$ & $3(1.9)$ \\
Candida spp. & $2(2.4)$ & $0(0.0)$ & $0(0.0)$ \\
Clostridium spp. & $2(2.4)$ & $1(2.8)$ & $3(1.9)$ \\
Corynebacterium spp. & $2(2.4)$ & $1(2.8)$ & $10(6.2)$ \\
Delftia spp. & $2(2.4)$ & $0(0.0)$ & $1(0.6)$ \\
Enterobacter spp. & $2(2.4)$ & $0(0.0)$ & $7(4.3)$ \\
Fusobacterium necrophorum & $2(2.4)$ & $0(0.0)$ & $0(0.0)$ \\
Micrococcus luteus & $2(2.4)$ & $1(2.8)$ & $3(1.9)$ \\
Stenotrophomonas maltophilia & $2(2.4)$ & $1(2.8)$ & $11(6.8)$ \\
Acinetobacter spp. & $1(1.2)$ & $1(2.8)$ & $1(0.6)$ \\
Actinomyces spp. & $1(1.2)$ & $0(0.0)$ & $1(0.6)$ \\
Dermabacter hominis & $1(1.2)$ & $0(0.0)$ & $0(0.0)$ \\
Histophilus somni & $1(1.2)$ & $0(0.0)$ & $0(0.0)$ \\
Lactobacillus agilis & $1(1.2)$ & $0(0.0)$ & $0(0.0)$ \\
Rothia amarae & $1(1.2)$ & $0(0.0)$ & $0(0.0)$ \\
Siccibacter turicensis & $1(1.2)$ & $0(0.0)$ & $57(35.4)^{\mathrm{a}}$ \\
No isolation & $15(18.1)^{\mathrm{b}}$ & $14(40.0)^{\mathrm{a}}$ &
\end{tabular}

${ }^{\mathrm{a}, \mathrm{b}}$ Values within a row with different superscript letters differ at $P<0.05$.

assignment, and scores $\geq 1.7$ and $\leq 2.0$ were used for genus identification.

\section{Statistical Analysis}

A chi-squared test was deployed to compare the percentage frequencies of bacteriological findings in healthy, CE, and SCE groups. In cases where the expected value was $\leq 5$, Fisher's exact test was used. The Venn diagram was constructed using an online tool http://bioinformatics.psb.ugent.be/webtools/Venn/ (Bioinformatics and Evolutionary Genomics Group, Ghent University).

\section{RESULTS}

The frequency of samples without bacterial isolation was $40 \%(14 / 35)$ in SCE and $35.4 \%(57 / 161)$ in healthy animals and greater than $18.1 \%(15 / 83)$ in the $\mathrm{CE}$ group $(P=0.009)$. A total of 127 bacterial species of 48 genera were identified in the 3 groups of animals (Table 1). Healthy animals presented numeri- cally greater richness (97 identified species) followed by the CE group (53 identified species), whereas only 21 bacterial species were identified among the SCE group. However, most of the species from the SCE group are shared between healthy animals and those with $\mathrm{CE}$ (Figure 1). Also, the bacterial species common to all 3 groups include the most prevalent microorganisms among studied animals, such as E. coli, Aerococcus viridans, and Bacillus cereus (Supplemental Table S1; Paiano et al., 2021). Interestingly, T. pyogenes was the most frequent pathogen among the CE group (32.4\%, $22 / 68)$, differing $(P<0.001)$ from the SCE and healthy groups, respectively.

Considering the healthy animals, isolates from the Staphylococcus genus were most frequently detected $(18.6 \%, 30 / 161)$, followed by isolates belonging to the genera Escherichia $(14.9 \%, 24 / 161)$, Bacillus $(12.4 \%$, 20/161), Enterococcus $(11.1 \%, 18 / 161)$, and Aerococcus $(10.6 \%, 17 / 161)$. For CE cows, the Trueperella genus was the most frequent $(26.5 \%, 22 / 83)$, followed by isolates belonging to the genera Escherichia (19.3\%, 16/83), Staphylococcus $(16.9 \%, 14 / 83)$, Bacillus (14.4\%, 


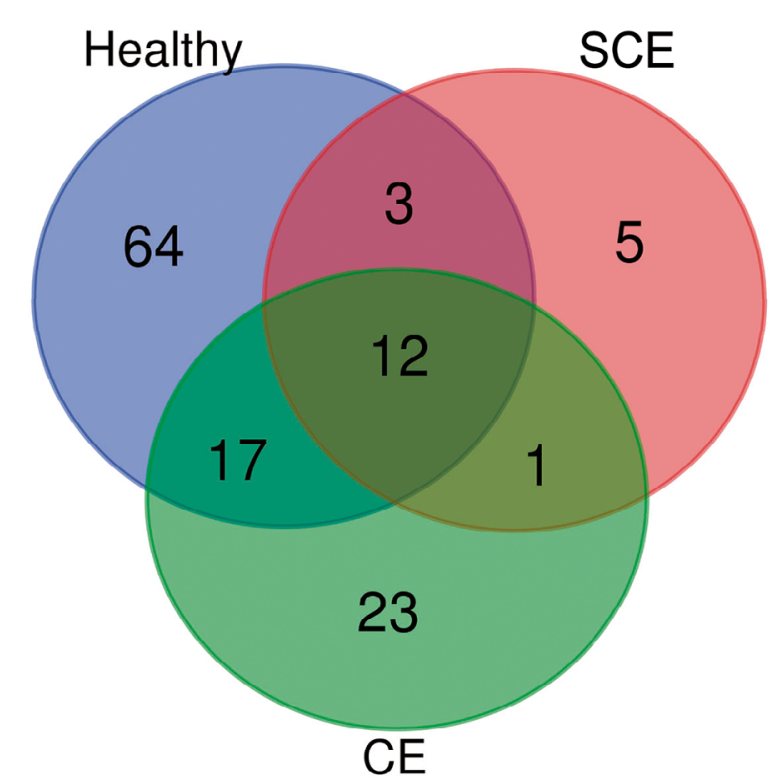

\begin{tabular}{l} 
CE \\
Number 23 \\
\hline Acinetobacter beijerinakii \\
Actinomyces hyovaginalis \\
Bacteroides pyogenes \\
Clostridium diolis \\
Dermabacter hominis \\
Enterococcus aerogenes \\
Enterococcus villorum \\
Fusobacterium necrophorum \\
Helcococcus kunzii \\
Helcococcus ovis \\
Histophilus somni \\
Klebsiella pneumoniae \\
Lactobacillus agilis \\
Lysinibacillus xylanilyticus \\
Paenibacillus residui \\
Paenibacillus turicensis \\
Porphyromonas levii \\
Prevotella heparinolytica \\
Pseudomonas aeruginosa \\
Rothia amarae \\
Streptococcus pneumoniae \\
Streptococcus salivarius \\
Streptococcus sanguinis
\end{tabular}

\begin{tabular}{l} 
Healthy $\times$ SCE \\
Number 3 \\
\hline Corynebacterium efficiens \\
Paenibacillus phoenicis \\
Staphylococcus chromogenes \\
CE x SCE \\
Number 1 \\
\hline Pseudomonas rhodesiae
\end{tabular}

\section{SCE}

\section{Number 5}

Acinetobacter radioresistens

Bacillus megaterium

Bacillus siralis

Scedosporium apiospermum

Streptococcus uberis

\section{CE $x$ Healthy}

Number 17

Bifidobacterium pseudolongum

Corynebacterium xerosis

Delftia acidovorans

Enterobacter cloacae

Enterococcus faecium

Enterococcus gallinarum

Enterococcus hirae

Klebsiella oxytoca

Lysinibacillus massiliensis

Paenibacillus macerans

Pseudomonas monteilii

Siccibacter turicensis

Staphylococcus arlettae

Staphylococcus epidermidis

Staphylococcus hominis

Staphylococcus xylosus

Trueperella pyogenes

\section{CE $x$ Healthy $\times$ SCE}

Number 12

Aerococcus viridans

Bacillus cereus

Bacillus licheniformis

Clostridium perfringens

Escherichia coli

Paenibacillus cookii

Pseudomonas fulva

Pseudomonas stutzeri

Staphylococcus warneri

Stenotrophomonas maltophilia

Streptococcus pluranimalium
Micrococcus luteus
Healthy

Number 64

Acinetobacter schindleri

Actinomyces odontolyticus

Atopobium minutum

Bacillus altitudinis

Bacillus clausii

Bacillus coagulans

Bacillus oleronius

Bacillus pumilus

Bacillus thuringiensis

Brachybacterium conglomeratum

Brevibacillus a gri

Brevibacillus laterosporus

Brevibacillus parabrevis

Brevibacterium luteolum

Cellulosimicrobium cellulans

Citrobacter amalonaticus

Citrobacter freundii

Citrobacter koseri

Corynebacterium flavencens

Corynebacterium glutamicum

Corynebacterium jeikeium

Corynebacterium stationis

Cutibacterium acnes

Enterobacter kobei

Enterobacter xiangfangensis

Enterococcus avium

Enterococcus casseliflavus

Enterococcus durans

Enterococcus faecal is

Enterococcus italicus

Enterococcus mundtii

Enterococcus saccarolyticus

Enterococcus thailandicus

Escherichia hermannii

Klebsiella variicola

Kocuria marina

Kocuria rhizophila

Leclercia adecarboxylata

Lysinibacillus fusiforms

Macrococcus canis

Microbacterium esteraromaticum

Microbacterium oxydans

Microbacterium paraoxydans

Micrococcus lylae

Paenibacillus a mynolyticus

Paenibacillus barcinonensis

Paenibacillus illinoisensis

Pantoea agglomerans

Pantoea ananatis

Pluralibacter gergoviae

Proteus hauseri

Pseudomonas putida

Roseomonas mucosa

Salmonella enterica

Slaphylococcus caprae

Staphylococcus sciuri

Staphylococcus cohnii

Staphylococcushaemolyticus

Streptococcus alactolyticus

Streptococcus dysgalactiae

Streptococcus lutetiensis

Streptococcus lutetiensis

Streptococcus mitis

Vagococcus fluvialis

Figure 1. Venn diagram illustrating the common and exclusive bacterial species isolated from the uterine samples collected of the 3 groups [healthy, clinical endometritis (CE), and subclinical endometritis (SCE)]. 
A

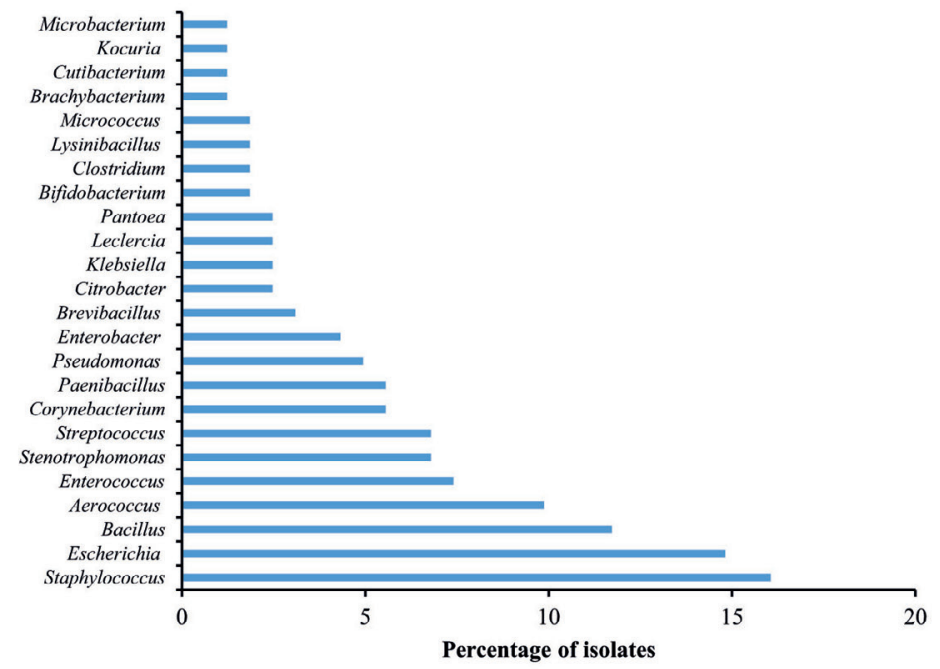

B

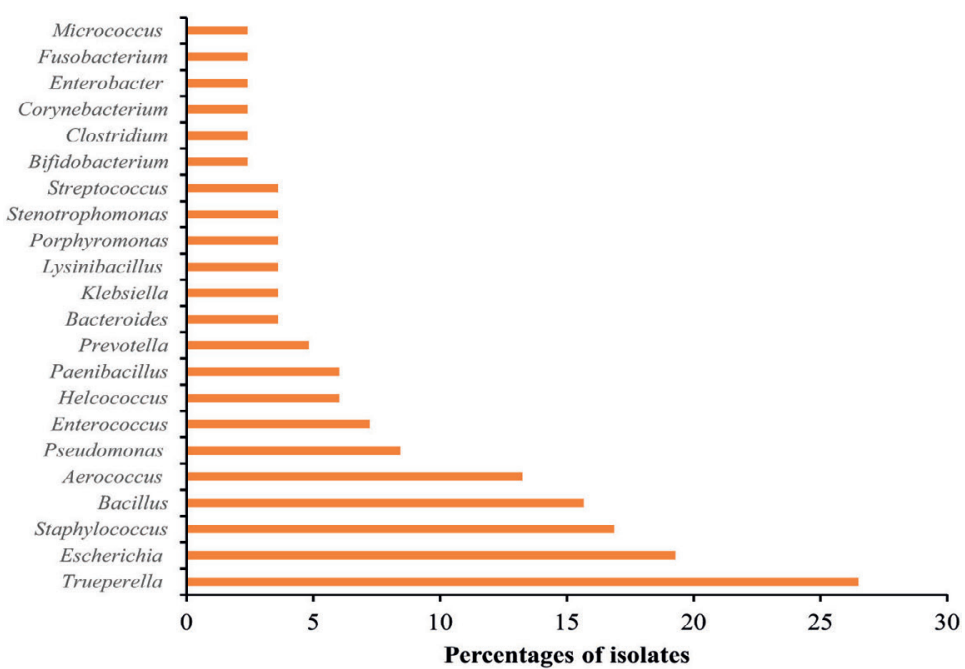

C

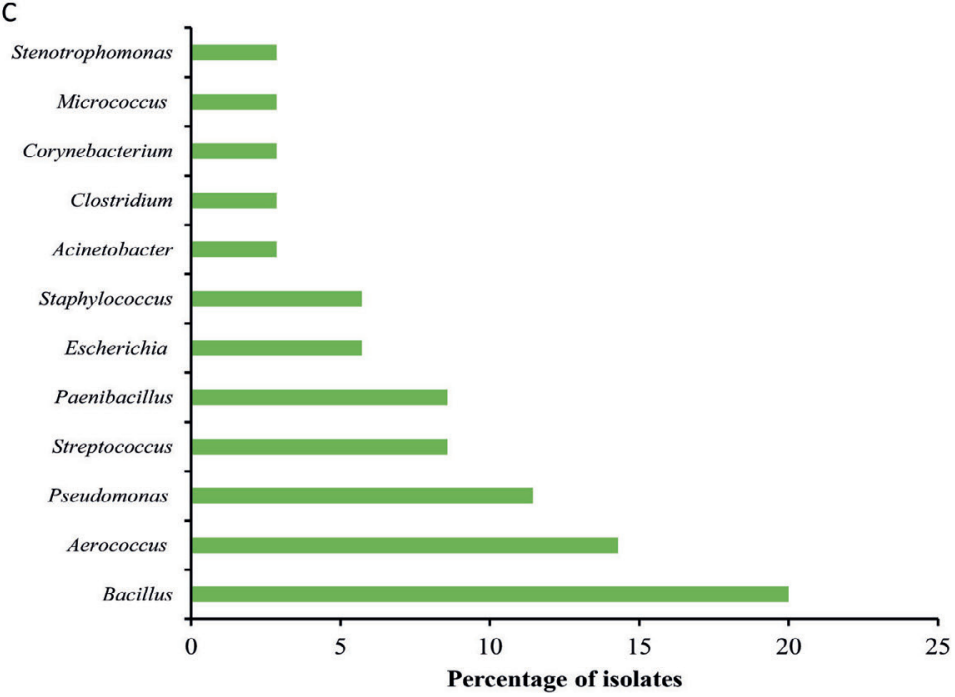

Figure 2. Frequency of different genera isolated from (A) healthy animals, (B) cows with clinical endometritis, and (C) cows with subclinical endometritis. 
12/83), Aerococcus (13.3\%, 11/83), and Pseudomonas $(8.4 \%, 7 / 83)$, respectively. Finally, concerning cows with SCE, isolates of the Bacillus genus were detected most frequently $(22.9 \%, 8 / 35)$, followed by the genera Aerococcus (14.3\%, 5/35), Pseudomonas (11.4\%, 4/35), Streptococcus $(8.6 \%, 3 / 35)$, and Paenibacillus (8.6\%, $3 / 35$ ), respectively (Figure 2).

The most frequent species belonging to the predominant genera for healthy, CE, and SCE cows are presented in Figure 3. This highlights the greater richness of the Enterococcus, Staphylococcus, and Bacillus genera. For the healthy cows, the genus Enterococcus represented the most varied group, including 11 different species, with Enterococcus faecium $(5 / 18,27.8 \%)$ being the most prevalent. Next, the Staphylococcus and Bacillus genera were composed of 10 and 8 different species, respectively, with a predominance of Staphylococcus xylosus $(11 / 30,36.7 \%)$ and B. cereus $(7 / 20,35.0 \%)$. Regarding cows with $\mathrm{CE}$, the genus Staphylococcus represented the most heterogeneous group, including 5 different species. Meanwhile, in the SCE group, the ge- nus Bacillus was the most diverse, including 4 different species with a predominance of $B$. cereus $(5 / 8,62.5 \%)$. Interestingly, for the Aerococcus genus, only $A$. viridans was identified and presented a high frequency among all studied groups (Supplemental Table S1; Paiano et al., 2021).

We observed a significantly higher isolation rate $(P$ $=0.00024)$ of $T$. pyogenes in the CE group $(26.5 \%$, $22 / 83)$ compared with healthy $(0.6 \%, 1 / 161)$ and SCE cows $(0.0 \%, 0 / 35)$. For other genera or species, no significant differences were found in the percentages between healthy, CE, and SCE groups. Other species, such as Prevotella heparinolytica, Porphyromonas levii, Fusobacterium necrophorum, Bacteroides pyogenes, and Helcococcus spp. were exclusively isolated in the CE group, although they presented lower frequencies. Interestingly, these bacteria were isolated in association with $T$. pyogenes. Of the total samples with $T$. pyogenes isolation $(\mathrm{n}=22)$, in 4 samples we observed this agent as a monoculture, 6 samples presented an association with $E$. coli, and in 12 samples the agent was associated

A
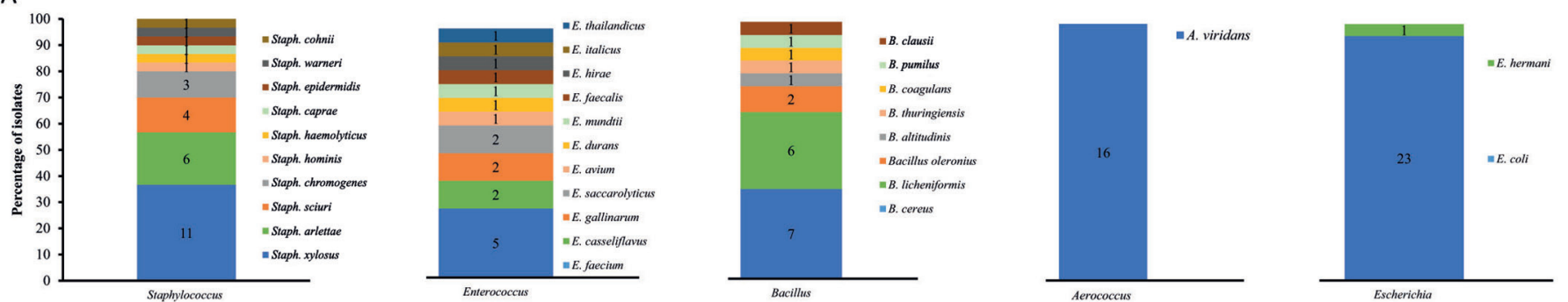

B
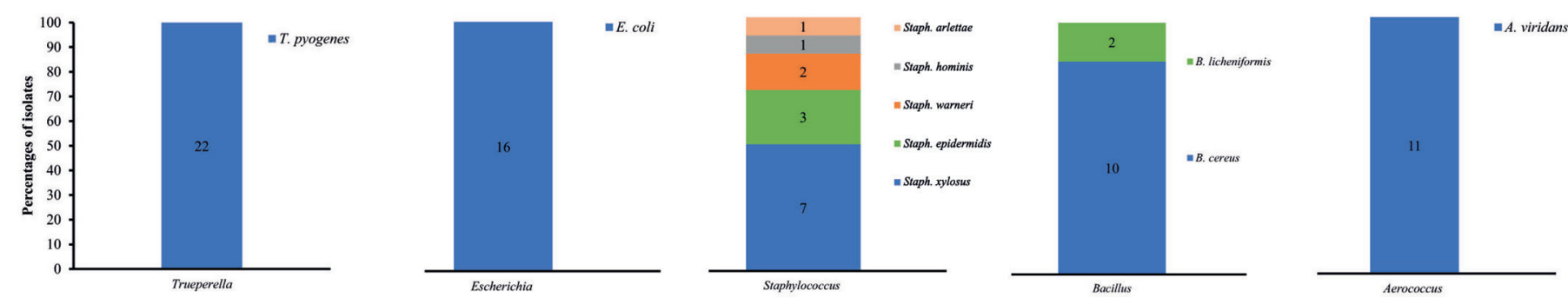

C
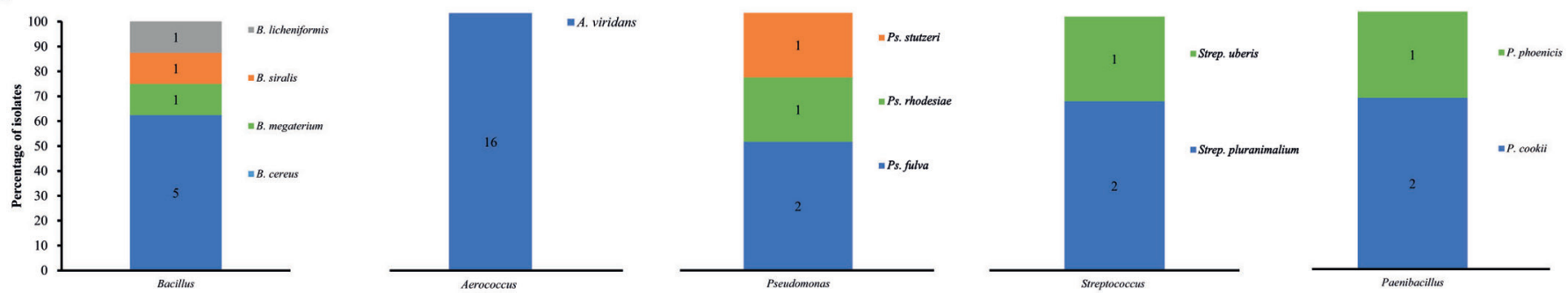

Figure 3. Most frequently isolated genera that were shown on the species level. Stacked bars represent the relative frequency of pathogens isolated from (A) healthy cows, (B) cows with clinical endometritis, and (C) cows with subclinical endometritis. The numbers within the stacked bars indicate the absolute number of isolates of each species. 
with different bacterial species (Supplemental Table S2; Paiano et al., 2021).

The number of bacterial species isolated according to the health status of animals is presented in Figure 4 and Supplemental Table S1 (Paiano et al., 2021), and the respective isolation profiles are described in Supplemental Tables S2 and S3 (Paiano et al., 2021). We found a predominance of monocultures for the healthy and SCE groups ( $42.3 \%$ and $61.9 \%$, respectively), whereas the cows with $\mathrm{CE}$ presented a high frequency of infections caused by 2 distinct bacterial species $(39.7 \%)$ and monocultures $(36.8 \%)$, respectively. Among the detected monocultures, $A$. viridans and $B$. cereus predominated among the 3 groups, and $E$. coli and Staph. xylosus were shared between the healthy and CE groups; however, T. pyogenes and Stenotrophomonas maltophilia and Streptococcus pluranimalium were exclusively detected in the CE and healthy cows, respectively (Supplemental Tables S2 and S3; Paiano et al., 2021). High heterogeneity of isolation profiles was observed for the healthy cows.

\section{DISCUSSION}

There is a growing interest in the microbiota of the reproductive tract of dairy cows. Studies in this field are fundamental for knowledge of the most frequent pathogens that can cause uterine diseases and affect the fertility of dairy cows. More studies will also contribute to new therapies focused exclusively on the main microorganisms associated with uterine diseases.

Previously, the uterus was believed to be sterile during pregnancy and contaminated with nonspecific bacteria after parturition. However, evidence from different studies now suggests that the uterus is not sterile, but specific microorganisms are adapted to the endometrium and can also influence the uterine cells present in the microenvironment. Modern culture-independent molecular methods focused on sequencing have also widened our present understanding of the microbiome of the uterus in cattle with metritis, pyometra, and endometritis (Appiah et al., 2020). Furthermore, the high percentage of healthy animals bacteriologically positive in our study supports the hypothesis that the healthy uterus is not sterile.

Our findings reveal a diverse community of 127 different species representing 48 genera. Among them, the most prevalent genera were Escherichia, Staphylococcus, Bacillus, and Aerococcus, and the most prevalent species were $E$. coli and A. viridans. The results of the present study are corroborated by Wagener et al. (2015), who reported a diverse uterine microbiota consisting of 202 different species and 76 genera, and Ballas et al. (2021), who identified 116 different species and 49 genera. Ballas et al. (2021) identified a high prevalence of bacteria of the Bacillus and Staphylococcus genera in cows with

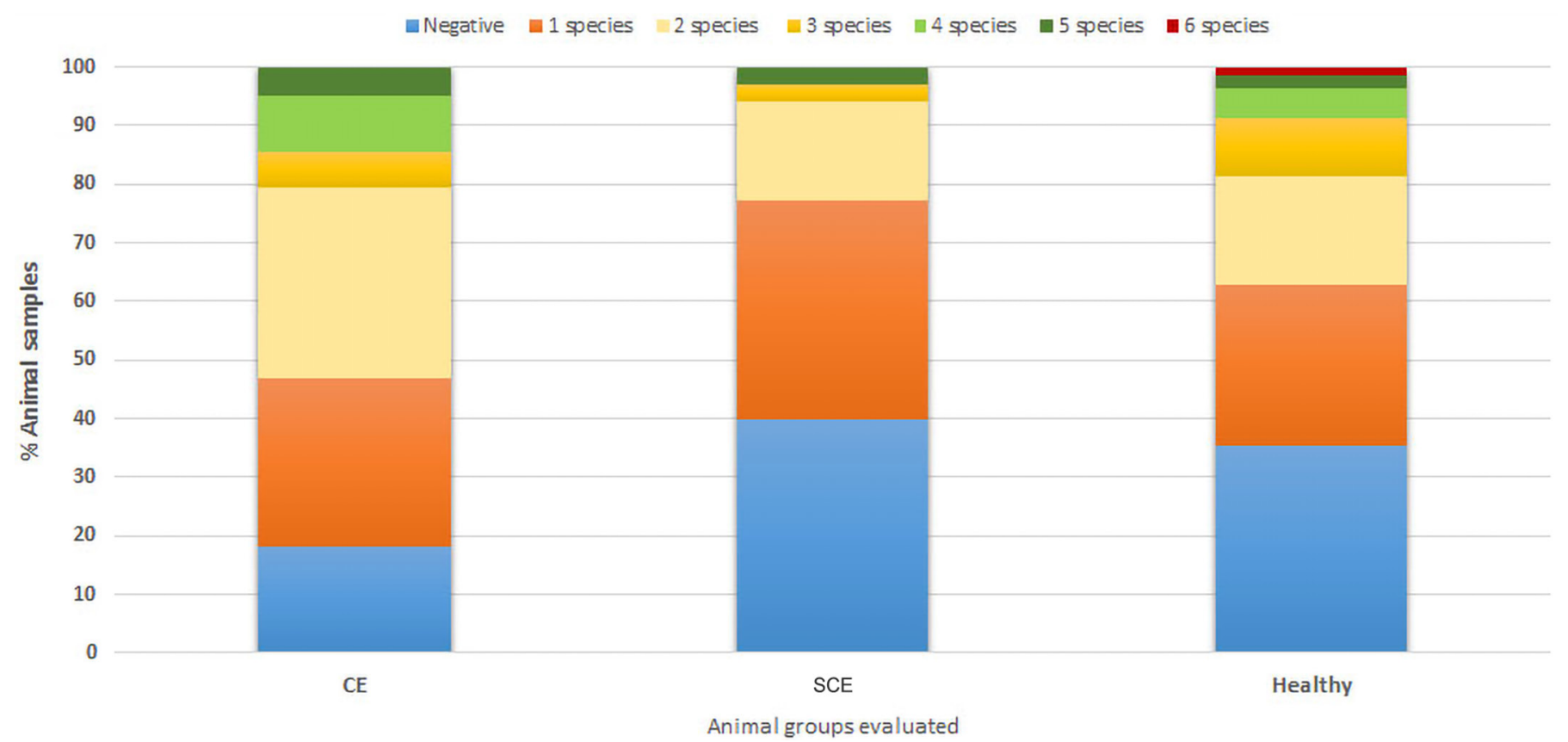

Figure 4. Frequency of bacterial species isolated according to groups of evaluated cows. Stacked bars represent the relative frequency of samples with 0 to 6 different bacterial species isolated in cows with clinical endometritis (CE), cows with subclinical endometritis (SCE), and healthy cows. 
and without endometritis at the time of AI in 120 dairy cows from Slovakia. However, the same authors identified a low prevalence of Trueperella and Escherichia in uterine isolates. In Austria, Wagener et al. (2015) reported Staphylococcus, Trueperella, and Escherichia as the most prevalent genera in cows with and without endometritis during the puerperium period in 122 evaluated cows.

Our study showed that the most frequent bacteria isolated from CE cows were T. pyogenes. These findings agree with previous studies conducted in Europe (Werner et al., 2012; Wagener et al., 2014). Using culture-independent methods, Bogado Pascottini et al. (2020) observed that cows with CE had microbiota characterized by a greater relative abundance of Fusobacterium and Trueperella and a lower relative abundance of Escherichia, Shigella, Lactobacillus, Prevotella, Schlegelella, Staphylococcus, and Streptococcus than healthy and SCE cows.

Among SCE cows, no T. pyogenes was isolated, corroborating the findings of Madoz et al. (2013), who did not identify $T$. pyogenes in uterine samples from cows diagnosed with SCE in Argentina. Moreover, Sens and Heuwieser (2013) highlighted that T. pyogenes does not seem to be the main key for cows to develop subclinical endometritis. Our observations corroborate previous studies (Wang et al., 2018), supporting that uterine infections with the main pathogens play a minor role in cows with SCE compared with CE cows. The uterine defense mechanism that occurs while the inflammatory response is still in progress can remove the intrauterine bacteria, restoring the normal uterine environment (Appiah et al., 2020). The establishment of uterine infections depends on the immune status of the animal and the pathogenicity of the invading microorganisms (Wang et al., 2018). Several factors can influence the pathogenicity of the microorganisms, including the bacterial load and virulence, and the interactions between species (Dadarwal et al., 2017).

Our findings also revealed that Prevotella heparinolytica, Porphyromonas levii, Fusobacterium necrophorum, Bacteroides pyogenes, and Helcococcus spp. were identified only in CE cows. In dairy herds in Canada, Bogado Pascottini et al. (2020) identified, at the genus level, that cows with $\mathrm{CE}$ had a higher prevalence of Helcococcus, Fusobacterium, Trueperella, and Porphyromonas than healthy cows, and Fusobacterium and Trueperella compared with SCE cows. In a commercial dairy farm in China, Wang et al. (2018) highlighted a positive correlation between Fusobacterium, Bacteroides, Porphyromonas, and Helcococcus, in addition to Trueperella, with CE in dairy cows, compared with healthy and SCE cows.
In the present study, the methods of sample collection, transportation, and culture media used permitted the isolation of a large diversity of aerobic and anaerobic agents, in addition to the limitations of culture-dependent techniques. Bogado Pascottini et al. (2020) found that the isolation of anaerobic agents was reduced compared with the abundance observed in metagenomic analysis. Those authors found that the number of samples collected and more controls in anaerobic culture could be important in their study. Our study demonstrated that the technique used for culture and identification of microorganisms present in the uterine environment made it possible to isolate a large diversity of bacterial species, aerobic and anaerobic. However, we cannot rule out the probability of false-negative results.

Regarding the reproductive performance of dairy cows affected by uterine diseases according to the presence of microorganisms in the uterine environment, the results of the literature are controversial. Sens and Heuwieser (2013) noted that cows with positive bacterial isolation had pregnancy at 250 DIM reduced by $19.9 \%$, and the days to pregnancy was increased by $75.5 \mathrm{~d}$ compared with cows with negative bacterial isolation. Werner et al. (2012) did not observe any difference in the pregnancy rate of cows with positive and negative bacterial isolation, but Gilbert and Santos (2016) observed that cows with positive bacterial isolation became pregnant earlier than cows with negative bacterial isolation. Denis-Robichaud and Dubuc (2015) demonstrated that intrauterine infusion of cephapirin improved the occurrence of first-service pregnancy in cows with $\mathrm{CE}$ and SCE. However, their study did not identify the bacteria present in the uterine environment.

This lack of agreement in the literature may occur due to the period after parturition in which the exams are performed, as well as the methodology used to collect, transport, and process the uterine samples to perform the bacterial isolation. Sens and Heuwieser (2013) highlighted that positive isolation of microorganisms does not seem to be the main key for cows to develop subclinical endometritis. We highlight that $18.1 \%$ and $40 \%$ of the samples from CE and SCE cows, respectively, did not present bacterial isolation.

Clinical and subclinical endometritis cause substantial impairment of reproductive performance in affected cows, but the economic benefit of efforts to identify and treat these individuals is herd specific (LeBlanc, 2008). Considering this information, it is very important to perform a deep evaluation of the herd's history and the reproductive effects observed, before determining the diagnostic tools to use and the control program to be adopted. 


\section{CONCLUSIONS}

The aerobic and anaerobic cultivable uterine bacterial community identified in this study was rich and diverse in healthy, SCE, and CE animals. Nevertheless, some bacteria, such as Prevotella heparinolytica, Porphyromonas levii, Fusobacterium necrophorum, Bacteroides pyogenes, and Helcococcus spp., were identified only in CE cows. Additionally, there was a significantly higher isolation rate of $T$. pyogenes in the $\mathrm{CE}$ group compared with healthy and SCE animals, reaffirming the importance of this pathogen in cases of endometritis.

\section{ACKNOWLEDGMENTS}

This study was financed in part by the Coordination of Improvement of Higher Education Personnel (CAPES, Brasilia, Brazil; Finance Code 001) and the National Council for Scientific and Technological Development (CNPq, Brasilia, Brazil). $\mathrm{AMM}$ is a $\mathrm{CNPq}$ fellow (grant no. 310736/2018-8). The authors have not stated any conflicts of interest.

\section{REFERENCES}

Appiah, M. O., J. Wang, and W. Lu. 2020. Microflora in the reproductive tract of cattle. Agriculture 10:232. https://doi.org/10.3390/ agriculture10060232.

Ballas, P., U. Reinländer, R. Schlegl, M. Ehling-Schulz, M. Drillich, and K. Wagener. 2021. Characterization of intrauterine cultivable aerobic microbiota at the time of insemination in dairy cows with and without mild endometritis. Theriogenology 159:28-34. https:/ /doi.org/10.1016/j.theriogenology.2020.10.018.

Bicalho, M. L., T. Santin, M. X. Rodrigues, C. E. Marques, S. F. Lima, and R. C. Bicalho. 2017a. Dynamics of the microbiota found in the vaginas of dairy cows during the transition period: Associations with uterine diseases and reproductive outcome. J. Dairy Sci. 100:3043-3058. https://doi.org/10.3168/jds.2016-11623.

Bicalho, M. L. S., S. Lima, C. H. Higgins, V. S. Machado, F. S. Lima, and R. C. Bicalho. 2017b. Genetic and functional analysis of the bovine uterine microbiota. Part II: purulent vaginal discharge versus healthy cows. J. Dairy Sci. 100:3863-3874. https://doi.org/10 $.3168 /$ jds.2016-12061.

Bogado Pascottini, O., J. F. W. Spricigo, S. J. Van Schyndel, B. Mion, J. Rousseau, J. S. Weese, and S. J. LeBlanc. 2021. Effects of parity, blood progesterone, and non-steroidal anti-inflammatory treatment on the dynamics of the uterine microbiota of healthy postpartum dairy cows. PLoS One 16:e0233943. https://doi.org/ 10.1371/journal.pone.0233943.

Bogado Pascottini, O., S. J. Van Schyndel, J. F. W. Spricigo, J. Rousseau, J. S. Weese, and S. J. LeBlanc. 2020. Dynamics of uterine microbiota in postpartum dairy cows with clinical or subclinical endometritis. Sci. Rep. 10:12353. https://doi.org/10.1038/s41598 -020-69317-z.

Dadarwal, D., C. Palmer, and P. Griebel. 2017. Mucosal immunity of the postpartum bovine genital tract. Theriogenology 104:62-71. https://doi.org/10.1016/j.theriogenology.2017.08.010.

Denis-Robichaud, J., and J. Dubuc. 2015. Randomized clinical trial of intrauterine cephapirin infusion in dairy cows for the treatment of purulent vaginal discharge and cytological endometritis. J. Dairy Sci. 98:6856-6864. https://doi.org/10.3168/jds.2014-9129.
Gilbert, R. O., and N. R. Santos. 2016. Dynamics of postpartum endometrial cytology and bacteriology and their relationship to fertility in dairy cows. Theriogenology 85:1367-1374. https://doi.org/ 10.1016/j.theriogenology.2015.10.045.

Gilbert, R. O., S. T. Shin, C. L. Guard, H. N. Erb, and M. Frajblat. 2005. Prevalence of endometritis and its effects on reproductive performance of dairy cows. Theriogenology 64:1879-1888. https:// doi.org/10.1016/j.theriogenology.2005.04.022.

Hijazin, M., J. Alber, C. Lammler, T. Weitzel, A. A. Hassan, M. Timke, M. Kostrzewa, E. Prenger-Berninghoff, and M. Zschock. 2012. Identification of Trueperella (Arcanobacterium) bernardiae by matrix-assisted laser desorption/ionization time-of-flight mass spectrometry analysis and by species-specific PCR. J. Med. Microbiol. 61:457-459. https://doi.org/10.1099/jmm.0.035774-0.

Kasimanickam, R., T. F. Duffield, R. A. Foster, C. J. Gartley, K. E. Leslie, J. S. Walton, and W. H. Johnson. 2004. Endometrial cytology and ultrasonography for the detection of subclinical endometritis in postpartum dairy cows. Theriogenology 62:9-23. https:// doi.org/10.1016/j.theriogenology.2003.03.001.

LeBlanc, S. J. 2008. Postpartum uterine disease and dairy herd reproductive performance: A review. Vet. J. 176:102-114. https://doi .org/10.1016/j.tvjl.2007.12.019.

LeBlanc, S. J., T. F. Duffield, K. E. Leslie, K. G. Bateman, G. P. Keefe, J. S. Walton, and W. H. Johnson. 2002. Defining and diagnosing postpartum clinical endometritis and its impact on 381 reproductive performance in dairy cows. J. Dairy Sci. 85:2223-2236. https://doi.org/10.3168/jds.S0022-0302(02)74302-6.

Machado, V. S., G. Oikonomou, M. L. S. Bicalho, W. A. Knauer, R. Gilbert, and R. C. Bicalho. 2012. Investigation of postpartum dairy cows' uterine microbial diversity using metagenomic pyrosequencing of the 16S rRNA gene. Vet. Microbiol. 159:460-469. https://doi.org/10.1016/j.vetmic.2012.04.033.

Madoz, L. V., M. J. Giuliodori, M. Jaureguiberry, J. Plöntzke, M. Drillich, and R. L. de la Sota. 2013. The relationship between endometrial cytology during estrous cycle and cutoff points for the diagnosis of subclinical endometritis in grazing dairy cows. J. Dairy Sci. 96:4333-4339. https://doi.org/10.3168/jds.2012-6269.

NRC. 2001. Nutrient Requirements of Dairy Cattle. 7 th rev. ed. Natl. Acad. Press.

Paiano, R. B., D. Birgel, J. Bonilla, and E. H. Birgel Junior. 2020a. Alterations in biochemical profiles and reproduction performance in postpartum dairy cows with metritis. Reprod. Domest. Anim. 55:1599-1606. https://doi.org/10.1111/rda.13815.

Paiano, R. B., D. B. Birgel, J. Bonilla, and E. H. Birgel Junior. 2020b. Evaluation of biochemical profile of dairy cows with metabolic diseases in tropical conditions. Reprod. Domest. Anim. 55:1219-1228. https://doi.org/10.1111/rda.13768.

Paiano, R. B., C. G. P. Gonçalves, J. P. G. Mendes, J. Bonilla, D. B. Birgel, and E. H. Birgel Junior. 2019. Comparative biochemical profiles, production and reproduction status of the postpartum dairy cows with and without purulent vaginal discharge. Reprod. Domest. Anim. 54:1188-1194. https://doi.org/10.1111/rda.13496.

Paiano, R. B., L. Z. Moreno, V. T. de M. Gomes, B. M. Parra, M. R. F. Barbosa, M. I. Z. Sato, J. Bonilla, G. Pugliesi, P. S. Baruselli, and A. M. Moreno. 2021. Bacteria isolated in healthy dairy cows uterus or in animals with clinical and subclinical endometritis (Version 1.1). Zenodo. https://doi.org/10.5281/zenodo.4702662

Prunner, I., K. Wagener, H. Pothmann, M. Ehling-Schulz, and M. Drillich. 2014. Risk factors for uterine diseases on small- and medium-sized dairy farms determined by clinical, bacteriological, and cytological examinations. Theriogenology 82:857-865. https://doi .org/10.1016/j.theriogenology.2014.06.015.

Sens, A., and W. Heuwieser. 2013. Presence of Escherichia coli, Trueperella pyogenes, $\alpha$-hemolytic streptococci, and coagulase-negative staphylococci and prevalence of subclinical endometritis. J. Dairy Sci. 96:6347-6354. https://doi.org/10.3168/jds.2013-6646.

Sheldon, I. M., G. Lewis, S. LeBlanc, and R. Gilbert. 2006. Defining postpartum uterine disease in cattle. Theriogenology 65:15161530. https://doi.org/10.1016/j.theriogenology.2005.08.021. 
Sheldon, I. M., A. N. Rycroft, B. Dogan, M. Craven, J. J. Bromfield, A. Chandler, M. H. Roberts, S. B. Price, R. O. Gilbert, and K. W. Simpson. 2010. Specific strains of Escherichia coli are pathogenic for the endometrium of cattle and cause pelvic inflammatory disease in cattle and mice. PLoS One 5:e9192. https://doi.org/10 .1371/journal.pone.0009192.

Thrusfield, M. 2005. Veterinary Epidemiology. 2nd ed. UK Blackwell Science Ltd.

Wagener, K., T. Grunert, I. Prunner, M. Ehling-Schulz, and M. Drillich. 2014. Dynamics of uterine infections with Escherichia coli, Streptococcus uberis and Trueperella pyogenes in post-partum dairy cows and their association with clinical endometritis. Vet. J. 202:527-532. https://doi.org/10.1016/j.tvjl.2014.08.023.

Wagener, K., I. Prunner, H. Pothmann, M. Drillich, and M. EhlingSchulz. 2015. Diversity and health status specific fluctuations of intrauterine microbial communities in postpartum dairy cows. Vet. Microbiol. 175:286-293. https://doi.org/10.1016/j.vetmic.2014.11 .017 .

Wang, M., M. Liu, J. Xu, L. An, J. Wang, and Y. Zhu. 2018. Uterine microbiota of dairy cows with clinical and subclinical endometritis. Front. Microbiol. 9:2691. https://doi.org/10.3389/fmicb.2018 .02691 .
Werner, A., V. Suthar, J. Plöntzke, and W. Heuwieser. 2012. Relationship between bacteriological findings in the second and fourth weeks postpartum and uterine infection in dairy cows considering bacteriological results. J. Dairy Sci. 95:7105-7114. https://doi .org/10.3168/jds.2012-5627.

Williams, E. J., D. P. Fischer, D. U. Pfeiffer, G. C. England, D. E. Noakes, H. Dobson, and I. M. Sheldon. 2005. Clinical evaluation of postpartum vaginal mucus reflects uterine bacterial infection and the immune response in cattle. Theriogenology 63:102-117. https: //doi.org/10.1016/j.theriogenology.2004.03.017.

\section{ORCIDS}

L. Z. Moreno ํㅡ https://orcid.org/0000-0003-0134-9741

B. M. Parra @ https://orcid.org/0000-0003-1320-7943

M. R. Barbosa ๑ https://orcid.org/0000-0002-7730-9247

G. Pugliesi ๑ https://orcid.org/0000-0001-5739-0677

P. S. Baruselli $\odot$ https://orcid.org/0000-0002-6773-4450

A. M. Moreno 으 https://orcid.org/0000-0002-3290-566X 